\title{
Comparison of Entonox and Transcutaneous Electrical Nerve Stim- ulation (TENS) in Labor Pain
}

\author{
S. Samadzadeh ${ }^{1}$, N. Rezavand ${ }^{2}$, M. Yari ${ }^{3}$, M. Rezaei ${ }^{4}$, H. Faizmahdavi ${ }^{5}$, and M. \\ Hematti $^{6}$ \\ ${ }^{1}$ Department of Physical medicine \& Rehabilitation, ${ }^{2,5}$ Maternity Research Center, ${ }^{3}$ Department of anesthesiology and critical care, \\ ${ }^{4}$ Department of Biostatistics and Epidemiology, ${ }^{6} \mathrm{MSC}$ statistics, Kermanshah University of Medical Sciences, Kermanshah Iran.
}

\begin{abstract}
One of the concerns among mothers for delivery is labor pain. There are various methods to decrease labor pain, of which epidural anesthesia is an effective one, but not all mothers agree to receive this invasive intervention. Transcutaneous electrical nerve stimulation (TENS) is an inexpensive, safe, and easily applicable method which can be used in patient control mode. To find proper noninvasive methods to pain relief during labor this study was conducted to compare TENS, Entonox and combination of them during the first phase of labor. In this RCT study, 120 pregnant women in the age range of 20-40 years, singleton pregnancy, at 37-42 weeks' gestation, at cervical dilatation of less than $4 \mathrm{~cm}$ in phase one of labor were selected and randomly divided into three groups including TENS, Entonox and combination group. Pain severity was recorded based on VAS at the time of presentation to the maternity unit, cervical dilatations of 4-6cm, 6$8 \mathrm{~cm}$, and $8-10 \mathrm{~cm}$ and demographic characterizations. There were no significant differences regarding to age, parity, and BMI between the three groups. Pain was less severe in combination group compared to other two groups at cervical dilations of $4-6 \mathrm{~cm}$ and $6-8 \mathrm{~cm}$. At cervical dilation of $8-10 \mathrm{~cm}$, VAS score was higher in Entonox group than in other two groups, but no significant difference existed between TENS and combination group. Duration of the first phase of labor was longer in TENS group than the other two groups. TENS is a non-invasive, safe, easy applicable and self-administered method with low rate of complications which can be used alone or in combination with other methods in relieving labor pain.

Journal of Medical and Biomedical Sciences (2017) 6(2), 11-16
\end{abstract}

Keywords: Transcutaneous Electric Nerve Stimulation, Entonox, Labor Pain

\section{INTRODUCTION}

One of the concerns among mothers who are preparing for delivery is labor pain. By applying proper methods to decrease labor pain, not only this concern can be somehow addressed, but also maternal and fetal complications will decrease as a result of decreased stress responses (Saghiri et al., 2008). It has been shown that labor pain plays a role in the development of posttraumatic stress syndromes after childbirth (Arendt and Tessmer-Tuck, 2013).

There are various techniques to decrease labor pain,

Correspondence: Mitra Yari, Associate Professor, Department of anesthesiology and critical care. Kermanshah University of Medical Sciences, Kermanshab Iran, Imam Reza Teaching hospital. Email: yarimitra@yahoo.com of which epidural anesthesia is an effective method with appropriate outcome in managing labor pain (Gomar and Fernandez, 2000), but not all mothers agree to receive this intervention. Furthermore, this method cannot be accessed in all maternity centers. It was determined that epidural analgesia associated with prolonged labor, use of oxytocin augmentation and increased incidence of instrumental vaginal delivery (Kukulu and Demirok, 2008; Simmons et al., 2012).

Another adverse effects related to epidural analgesia are hypotension, motor blockade, fever and urinary retention(Jones et al., 2012). Also it has been shown that epidural anesthesia has a negative impact on breast-feeding in the first 24 hours of life (Baumgarder et al., 2003). Among other interven- 


\section{Noninvasive methods for relieving pain in labor}

Samadzadeh et al.,

tions to relieve labor pain are pharmacologic agents such as narcotics, hypnotics, Entonox, and nonpharmacologic methods like transcutaneous electrical nerve stimulation (TENS) (Habanananda, 2004; Miller, 2010; Jones et al., 2012).

Entonox is a gas mixture of oxygen $(50 \%)$ and nitrous oxide $(50 \%)$ which is inhaled through a oneway valve by mask during inspiration. Labor relief which is achieved by Entonox is associated without decrease in consciousness level. As it can be used in patient control mode, this method has gained much attention in labor. Intermittent use of Entonox is not associated with any complications and it is exhaled rapidly by expiration (1970; Baskett and Bennett, 1971; Latto et al., 1973; Pazandeh et al., 2004).

TENS is a non-invasive, inexpensive, safe, and easily applicable method which can be used in patient control mode. In this method, electricity is delivered through the skin causing stimulation of peripheral nerves and yielding a rapid relief in pain. It is used in both acute and chronic painful conditions (Augustinsson et al., 1977; Robson, 1979; Nesheim, 1981; van der Spank et al., 2000). There is no evidence that TENS causes any harm to mother and baby(Cluett, 1994).

Fibers which related to the transmission of pain are A-delta and $\mathrm{C}$ fibers which are thin fibers with high threshold. TENS stimulates A-beta fibers which are thick fibers with low threshold and are related to touch sensation. Based on gate-control theory, stimulation of A-beta fibers inhibits transmission of action potential to A-delta and $\mathrm{C}$ fibers in the dorsal horn of the spinal column. This, in turn, inhibits transmission of pain to brain centers. In addition, TENS has an inhibitory mechanism and also releases endogenous opioids (Robson, 1979). To find proper noninvasive methods for relieving pain during labor, this study was conducted to compare TENS, Entonox and combination of them during the first phase of labor.

\section{MATERIALS AND METHODS} Study design and population

This study is registered on IRCT.ir (Ref. No.
IRCT201011295274N1) as a randomized clinical trial (RCT). In this study, 120 pregnant women in the age range of $20-40$ years, singleton pregnancy, at 37-42 weeks' gestation, fetal vertex presentation, cervical dilatation of less than $4 \mathrm{~cm}$, and in phase one of labor who did not request epidural anesthesia were included. Exclusion criteria were multifetal pregnancy, cardiac diseases of mother, having pace maker, skin infectious diseases, and epilepsy.

\section{Data collection}

After obtaining informed consent and providing explanations about Entonox and TENS, the patients were randomly divided (by method of 1:1:1) into three groups. First group was TENS group (40 subjects) for whom pure oxygen was applied instead of Entonox and electrode pads were attached on T10-11 and S2-S4 dermatomes on both sides of the spinal column. Electrodes were attached and the TENS machine turns $\mathrm{ON}$ while a continuous frequency of $50 \mathrm{~Hz}$ was applied. During uterine contractions, mother was supposed to push the button $\mathrm{ON}$ resulted in burst stimulation with $2-\mathrm{Hz}$ frequency and $0.25 \mathrm{~ms}$ duration. The machine used was TENS (TENSMED, Netherland, Enrofnonius, 911) which measured $70 * 70 \mathrm{~mm}$. The electrode pads measured $90 * 32 \mathrm{~mm}$.

The second group (40 subjects) was Entonox group for whom similar to the first group; electrodes were placed on both sides of the spinal column and TENS machine was ON, but electrodes were not attached to the machine and Entonox was inhaled via mask during uterine contractions. Third group was combination group (40 subjects) in which both Entonox and TENS were applied.

In case of severe pain (VAS > 5), 25mg of meperidine was administered intravenously. Pain severity was recorded using visual analog scale (VAS) at the time of presentation to the maternity unit, and at cervical dilatations of $4-6 \mathrm{~cm}, 6-8 \mathrm{~cm}$, and $8-10 \mathrm{~cm}$. The VAS used here was a $10-\mathrm{cm}$ scale with 0 as no pain status and 10 as the most severe pain represented on the scale. Other variables recorded were age, parity, gestational age, BMI, duration of the first phase of labor from cervical dilation of $4 \mathrm{~cm}$, 
Noninvasive methods for relieving pain in labor Samadzadeh et al,

the amount of meperidine that used, and nausea and vomiting frequency.

\section{Statistical analysis}

Analysis of qualitative data was done by Chi-squared test and one-way logistic regression. Analysis of continuous variables were done by the KolmogorovSmirnov test, The Kruskal-Wallis test, and two-bytwo comparisons. $\mathrm{P}$ values of less than 0.05 were considered statistically significant.

\section{RESULTS}

There were no significant differences with regard to age, parity, and BMI between the three groups
(Table 1). Pain was less severe in combination group compared to other two groups at cervical dilations of $4-6 \mathrm{~cm}$ and $6-8 \mathrm{~cm}$. It should be noted that at the mentioned cervical dilations VAS score was higher in Entonox group than in TENS group, but the difference was not statistically significant.

Nausea frequency was different between the three groups and one-way logistic regression analysis demonstrated that the incidence of nausea in TENS and Entonox groups was respectively 2 and 5 times higher than in combination group $(\mathrm{P}=0.004)$ (Table 2).

Table 1. Demographic and Patients Characteristics of Patient Population

\begin{tabular}{lcccc}
\hline Demographic data & $\begin{array}{c}\text { Entonox group } \\
\text { No }=\mathbf{4 0}\end{array}$ & $\begin{array}{c}\text { TENS group } \\
\text { No }=\mathbf{4 0}\end{array}$ & $\begin{array}{c}\text { Combined group } \\
\text { No }=\mathbf{4 0}\end{array}$ & P value \\
\hline Age (year) & $26.68 \pm 6.49$ & $25.9 \pm 5.84$ & $29.31 \pm 6.41$ & 0.085 \\
Parity (frequency) & $1.9 \pm 1.6$ & $1.8 \pm 1.2$ & $1.9 \pm 1$ & 0.893 \\
BMI $\left(\mathrm{Kg} / \mathrm{m}^{2}\right)$ & $25.8 \pm 3.9$ & $24.6 \pm 3.9$ & $24.9 \pm 3.2$ & 0.126 \\
\hline
\end{tabular}

Data presented as Mean $\pm S D$, p-value $<0.05$ is statistically significant; BMI-body mass index

Table 2. Comparison of Pain Severity in all Groups of Study

\begin{tabular}{lll}
\hline $\begin{array}{l}\text { Uterine group Cervical } \\
\text { Dilatation }\end{array}$ & Mean (SD) & P value \\
\hline $4-6 \mathrm{~cm}$ & & \\
TENS & $3.60 \pm 1.44$ & 0.039 \\
Entonox & $3.85 \pm 1.91$ & \\
Combined & $2.93 \pm 1.42$ & \\
6-8cm & & \\
TENS & $4.50 \pm 2.14$ & 0.019 \\
Entonox & $4.93 \pm 2.46$ & \\
Combined & $3.55 \pm 1.37$ & \\
8-10cm & & \\
TENS & & \\
Entonox & $4.73 \pm 2.11$ & 0.010 \\
Combined & $6.23 \pm 3.02$ & \\
\hline
\end{tabular}

Data presented as Mean $\pm S D$, p-value $<0.05$ is statistically significant
At cervical dilation of $8-10 \mathrm{~cm}$, VAS score was higher in Entonox group than in other two groups, but no significant difference existed between TENS and combination group $(6.5,5$, and 4.3 respectively in Entonox, TENS, and combination groups) (Table 3).

The duration of first phase of labor was longer in TENS group (300.1 224.8) than other two groups and not significantly difference between combination (214.6 151.4) and Entonox group (228.5 92.4). Regarding the amount of analgesic administered, a significant difference was found between the three groups $(\mathrm{P}<0.001)$. According to the Chi-squared test, no significant difference existed between Entonox and TENS groups $(\mathrm{P}=0.066)$, but this variable was significantly lower in combination group compared to other two groups $(\mathrm{P}<0.001)$.

Table 3. Results from Intervention in all Groups of Study

\begin{tabular}{lllll}
\hline Results & Entonox, $\mathbf{n}(\mathbf{\%})$ & TENS, $\mathbf{n}(\mathbf{\%})$ & Combined, $\mathbf{n}(\mathbf{\%})$ & P value \\
\hline Nausea and vomiting & $17(42.5)$ & $17(42.5)$ & $5(20)$ & 0.004 \\
Amount of meperidine $(\mathrm{mg})$ & $34(85)$ & $27(67.5)$ & $12(30)$ & $<0.001$ \\
\hline
\end{tabular}

Data presented as Mean $\pm S D$, p-value $<0.05$ is statistically significant; BMI-body mass index 


\section{Noninvasive methods for relieving pain in labor}

Samadzadeh et al.,

\section{DISCUSSION}

Effectiveness of Entonox and TENS in decreasing pain were similar at the initial and middle stages of phase one of labor as pain was not severe, but influence of applying both methods were more significant in pain relief. At the end of labor and more cervical dilatations, using TENS alone or in combination with Entonox were more effective in relieving pain than using of Entonox alone.

Harrison et al. compared the analgesia induced by epidural anesthesia, Entonox, TENS, and intravenous narcotic during labor. They indicated that effective analgesia was achieved in $88 \%$ of mothers who underwent epidural anesthesia and partial effectiveness was observed in other methods. They also pointed out that Entonox was more effective in women who presented in earlier stages of labor (Harrison et al., 1987).

Chia et al. also compared analgesia induced by TENS and Entonox in labor and observed that these two methods had similar effect in decreasing labor pain and the best time that these exert their analgesic effect is when interval time between uterine contractions is 5-10 minutes (Chia et al., 1990).

Augustinson and colleagues studied the efficacy of TENS in labor pain and advocated TENS as a safe and beneficial method in decreasing labor pain. They proposed TENS could be used as a basic method for labor pain management without any side effect and the other methods can be added to this method for more efficacy. This is supported by our results since TENS is helpful in final period of the first phase of labor when labor pain is aggravated more (Augustinsson et al., 1977).

Kaplan et al. made use of TENS for pain management in the first phase of labor in 104 pregnant women (both primiparous and multiparous women). Most patients stated that TENS is effective in labor pain and asked to repeat this method in their future pregnancy and labor. They also reported that duration of the first phase of labor was shorter in the group for which TENS was applied compared to control group (Kaplan et al., 1998).
However, in the current study, TENS subjects did not have shorter first phase of labor. This discrepancy could be justified by the fact that in Kaplan's study, the control group had no pain, but in our study TENS group was compared to Entonox group and presumably Entonox was a factor which contributed to shortening of the first phase of labor. As seen in Harrison's study, where Entonox, TENS, epidural anesthesia, and intravenous medications were compared, it was reported that Entonox shortened the first phase of labor which is compatible with our results (Harrison et al., 1987). Tashani et al. also reported that TENS is a proper method for pain management in labor and in interpretation of mechanisms involved in pain relief stated that this method not only inhibits sending of signals through the dorsal horn to the brain, but also it causes release of endorphins and other neurochemicals such as 5HT, GABA, acetylcholine, adrenaline, and adenosine (Tashani and Johnson, 2009).

Stewart et al. stated that TENS has moderate efficacy in relieving labor pain (Stewart, 1979). But Vander et al. studied the effect of TENS on epidural anesthesia request in mothers, concluded that TENS had no effect on epidural anesthesia requests (van der Spank et al., 2000).This conclusion can be based on the fact that TENS can only induce a partial pain relief and compared to epidural anesthesia which has complete analgesia, TENS was not so demanded.

Nesheim et al. also indicated that TENS is not effective in labor pain relief (Nesheim, 1981). Van der ploeg et al. concluded that TENS was not more effective than a placebo during the first stage of labor (van der Ploeg et al., 1996). Bedwell et al. supporting a review of evidence on the effectiveness of TENS for pain relief in labor, stated that there is only limited evidence that TENS reduces pain in labor (Bedwell et al., 2011).

Tsen et al. made use of TENS in combination with CSE(combined spinal epidural) and concluded that TENS had no effect on lengthening the duration of analgesia by CSE (Tsen et al., 2001). All of these 


\section{Noninvasive methods for relieving pain in labor}

Samadzadeh et al.,

studies emphasized that TENS has a partial analgesic effect in labor pain and in comparison to CSE and epidural analgesia has less analgesic effect. Regarding to medication, we concluded that analgesic was less frequently requested in combination group and no significant difference was found between Entonox and TENS groups. Nesheim et al. did not report a decrease in analgesic use when compared TENS to control group (Nesheim, 1981).

Harrison et al. stated that in their study, requirement for analgesia was similar in TENS and Entonox groups (Harrison et al., 1987) which is consistent with our results and demonstrates the cumulative effect of using two methods for pain control at the same time. According to the obtained findings, nausea and vomiting was more common in Entonox group compared to TENS group, but this was lower in combination group than in other groups. This may indicate relationship between pain and occurrence of nausea and vomiting owing to the fact that there was less nausea in the combination group. It is likely that lower rate of nausea and vomiting in combination group correlates with less use of narcotics in this group. It is suggested to do a study in the future to investigate the relationship between Entonox and occurrence of nausea and vomiting.

\section{CONCLUSION}

Transcutaneous electrical nerve stimulation (TENS) which is serve as non-invasive, safe, easy applicable and self-administered method with low rate of complications can be used alone or in combination with other methods in relieving labor pain.

\section{ACKNOWLEDGMENT}

We thank Dr. FarivarFerdowsi, resident of obstetrics and gynecology who helped in gathering the data and also Ms. Almasi for her sincere help.

\section{COMPETING INTERESTS}

The authors declare that they have no competing interests.

\section{REFERENCES}

(1970) Clinical trials of different concentrations of oxygen and nitrous oxide for obstetric anal- gesia. Report to the Medical Research Council of the Committee on Nitrous Oxide and Oxygen Analgesia in Midwifery. $\mathrm{Br}$ Med J 1(5698), 709-713.

Arendt K.W. and Tessmer-Tuck J.A. (2013) Nonpharmacologic labor analgesia. Clin Perinatol 40(3), 351-371.

Augustinsson L.E., Bohlin P., Bundsen P., Carlsson C.A., Forssman L., Sjoberg P. and Tyreman N.O. (1977) Pain relief during delivery by transcutaneous electrical nerve stimulation. Pain 4(1), 59-65.

Baskett P.J. and Bennett J.A. (1971) Pain relief in hospital: the more widespread use of nitrous oxide. Br Med J 2(5760), 509-511.

Baumgarder D.J., Muehl P., Fischer M. and Pribbenow B. (2003) Effect of labor epidural anesthesia on breast-feeding of healthy fullterm newborns delivered vaginally. $J A m$ Board Fam Pract 16(1), 7-13.

Bedwell C., Dowswell T., Neilson J.P. and Lavender T. (2011) The use of transcutaneous electrical nerve stimulation (TENS) for pain relief in labour: a review of the evidence. Midwifery 27(5), e141-148.

Chia Y.T., Arulkumaran S., Chua S. and Ratnam S.S. (1990) Effectiveness of transcutaneous electric nerve stimulator for pain relief in labour. Asia Oceania J Obstet Gynaecol 16(2), 145-151.

Cluett E. (1994) Analgesia in labour: a review of the TENS method. Prof Care Mother Child 4(2), 50-52.

Gomar C. and Fernandez C. (2000) Epidural analgesia-anaesthesia in obstetrics. Eur J Anaesthesiol 17(9), 542-558.

Habanananda T. (2004) Non-pharmacological pain relief in labour. J Med Assoc Thai 87 Suppl 3S194-202.

Harrison R.F., Shore M., Woods T., Mathews G., Gardiner J. and Unwin A. (1987) A comparative study of transcutaneous electrical nerve stimulation (TENS), entonox, pethidine + promazine and lumbar epidural for pain relief in labor. Acta Obstet Gynecol Scand 66(1), 9-14. 


\section{Noninvasive methods for relieving pain in labor}

Samadzadeh et al.,

Jones L., Othman M., Dowswell T., Alfirevic Z., Gates S., Newburn M., Jordan S., Lavender T. and Neilson J.P. (2012) Pain management for women in labour: an overview of systematic reviews. Cochrane Database Syst Rev 3CD009234.

Kaplan B., Rabinerson D., Lurie S., Bar J., Krieser U.R. and Neri A. (1998) Transcutaneous electrical nerve stimulation (TENS) for adjuvant pain-relief during labor and delivery. Int J Gynaecol Obstet 60(3), 251-255.

Kukulu K. and Demirok H. (2008) Effects of epidural anesthesia on labor progress. Pain Manag Nurs 9(1), 10-16.

Latto I.P., Molloy M.J. and Rosen M. (1973) Arterial Concentrations of Nitrous Oxide during Intermittent Patient-Controlled Inhalation of 50\% Nitrous Oxide in Oxygen (Entonox) during the First Stage of Labour. British J Anaesth 45(10), 1029-1034.

Miller R.D. (2010) Text book. Anesthesia. Philadelphia: Churchil Livingstone.

Nesheim B.I. (1981) The use of transcutaneous nerve stimulation for pain relief during labor. A controlled clinical study. Acta Obstet Gynecol Scand 60(1), 13-16.

Pazandeh F., Firouzehchian F., Sharafi M. and Alavi Majd H. (2004) Comparison of inhalation of a mixture of oxygen and nitrous oxide with transcutaneous electrical nerve stimulation on the severity of pain in the active phase of labor among those referring to Tehran's Mahdieh maternity hospital in 2001. Pejouhesh 28(4), 269-273.

Robson J.E. (1979) Transcutaneous nerve stimulation for pain relief in labour. Anaesthesia 34 (4), 357-360.

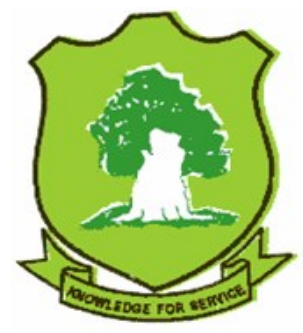

Saghiri M., Sattarzadeh N., Tabrizi N. and Pezeshki Z. (2008) A Comparative Study on the Severity of Labor Pain with or without Entonox and it's effects on the new-borns of Primiparas. I Ardabil Uni Med Sci 8(1), 6267.

Simmons S.W., Taghizadeh N., Dennis A.T., Hughes D. and Cyna A.M. (2012) Combined spinal-epidural versus epidural analgesia in labour. Cocbrane Database Syst Rev 10CD003401.

Stewart P. (1979) Transcutaneous nerve stimulation as a method of analgesia in labour. Anaesthesia 34(4), 361-364.

Tashani O. and Johnson M. (2009) Transcutaneous Electrical Nerve Stimulation (TENS) A Possible Aid for Pain Relief in Developing Countries? Libyan J Med 4(2), 62-65.

Tsen L.C., Thomas J., Segal S., Datta S. and Bader A.M. (2001) Transcutaneous electrical nerve stimulation does not augment epidural labor analgesia. J Clin Anesth 13(8), 571-575.

van der Ploeg J.M., Vervest H.A., Liem A.L. and Schagen van Leeuwen J.H. (1996) Transcutaneous nerve stimulation (TENS) during the first stage of labour: a randomized clinical trial. Pain 68(1), 75-78.

van der Spank J.T., Cambier D.C., De Paepe H.M., Danneels L.A., Witvrouw E.E. and Beerens L. (2000) Pain relief in labour by transcutaneous electrical nerve stimulation (TENS). Arch Gynecol Obstet 264(3), 131136. 